\title{
Eddy current corrosion measurement of steel
}

\begin{abstract}
Contactless eddy current sensors allow efficient detection and measurement of the corrosion of metals (like carbon steel), also under the paint layer, as demonstrated in the described experiments and analysis. The corrosion was estimated by a Dodd-Deeds eddy current air-core single-coil model, according to the variation of the impedance in the sensor coil. In the case of magnetic materials (like carbon steel) the sensor signal depends not only on the conductivity of the specimen, but also on the magnetic permeability. Furthermore, the sensor lift-off influences the results, so appropriate lift-off compensation schemes are beneficial. In the current study a planar sensor coil and another ferrite-core probe were used. The estimated resolution of the corrosion layer measurement is about $1 \mu \mathrm{m}$ (or better). A PC-based application frontend for estimation of the corroded area was developed.
\end{abstract}

Keywords: eddy current, NDT, Dodd-Deeds model, corrosion measurement, carbon steel

\section{Introduction}

Eddy current sensors are widely used for non-destructive testing and characterization of metals and other electrically conductive materials [1]. The analysis of the complex impedance of the single-air-core measurement coil as a probe above the metal under test has been proposed in the works of Dodd and Deeds from the 1960s [2, 3]. Alternatively, two-coil measurement setups, where one coil is transmitting the AC signal and the other is receiving, have been proposed, in one case as the receiving sensor coil is on the same side of the metal plate ("reflection-type measurement") [4] or on the other side of the metal plate under test ("through-the-metal setup") [5]. Anyway, in the current work, the single-coil configuration [2] was used, as being simple and well matching with the theory in the wide frequency range (up to $10 \mathrm{MHz}$ ) [6-8]. In [9], a PCbased simulation tool has been demonstrated for solving the Dodd-Deeds model for one- or two-layer metal plates with measurement coil above the metal plate. An im-

\footnotetext{
Acknowledgement: The work described in this chapter has been supported by institutional grant IUT19-11 of the Estonian Research Council and the European Regional Development Fund supporting the Estonian Center of Research Excellence EXCITE and Competence Centre ELIKO.
}

Olev Märtens, Raul Land, Mart Min, Thomas Johann Seebeck Department of Electronics, Tallinn University of Technology, Tallinn, Estonia, e-mail: olev.martens@ttu.ee

Marek Rist, Marju Ferenets, Andres Käsper, Eliko Competence Centre, Tallinn, Estonia

https://doi.org/10.1515/9783110558920-012 
proved, computationally faster "series expansions" (instead of infinite integration in the space) version of the single-coil model is also available [10]. The Dodd-Deeds model and other similar algorithms solve the "forward problem" - that means, how the coil impedance depends on the parameters of the measured object (e.g., metal plate) and the measurement setup (parameters of the measurement coil). But in real life the solving of the inverse problem is needed - calculation of the properties (electrical conductivity and/or magnetic permeability and sensor coil lift-off) of the object under test from the measured complex impedance of the measurement coil. The lift-off effect and compensation of it have been discussed in [11]. Full inverse solutions have been proposed, e. g., in [12, 13]. One possible problem in eddy current measurements is the dependency of the results not only on the electrical conductivity of the material under test, but also on the magnetic permeability [14].

Some ideas on how to solve both these variables have been shown in $[15,16]$. In [17] it has been shown (for the carbon steel AISI 1018) that the magnetic permeability is a frequency-dependent complex-valued variable. The eddy current corrosion process investigation has been done in $[18,19]$. In [18] it has been shown that both the electrical conductivity and magnetic permeability of the corrosion area are much (by an order) smaller than the values for the carbon steel (for SS400 in their example).

\section{Research performed}

\subsection{Measurement setup with air-core coil}

The single-coil measurement setup is shown in Fig.1. The setup includes the planar (PCB) measurement coil (shown in Fig. 2) with internal and external diameters D1 $(2.5 \mathrm{~mm})$ and D2 $(16 \mathrm{~mm})$, height $h(20 \mu \mathrm{m})$, number of turns $N=50$ and lift-off $z$ and the metal (steel) plate with thickness of $d$ (about $2 \mathrm{~mm}$ in the described experiments). The expected electrical conductivity of the material is about $6 \mathrm{MS} / \mathrm{m}$. The impedance measurement instrument Wayne-Kerr 6500B has been used to get the coil impedance and a specially designed 3D scanner moving the probe (coil) has been developed (Fig. 3). Also the Windows-based software has been developed for reverse

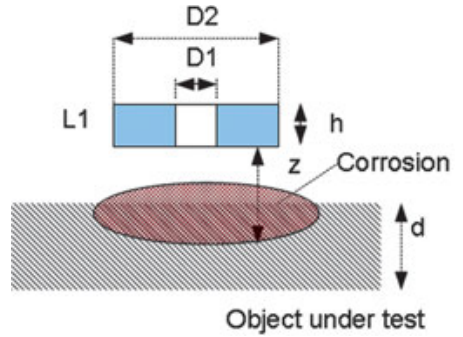

Fig. 1: Single-coil measurement setup. 

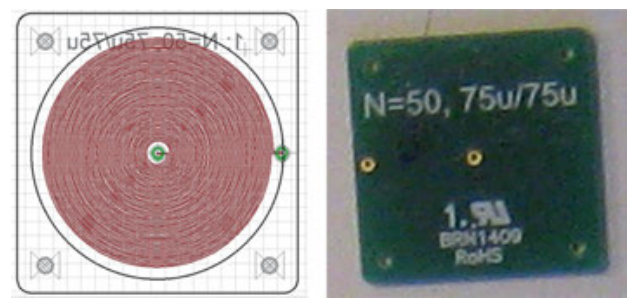

Fig. 2: Measurement coil - a photo (on the left) and PCB layout.

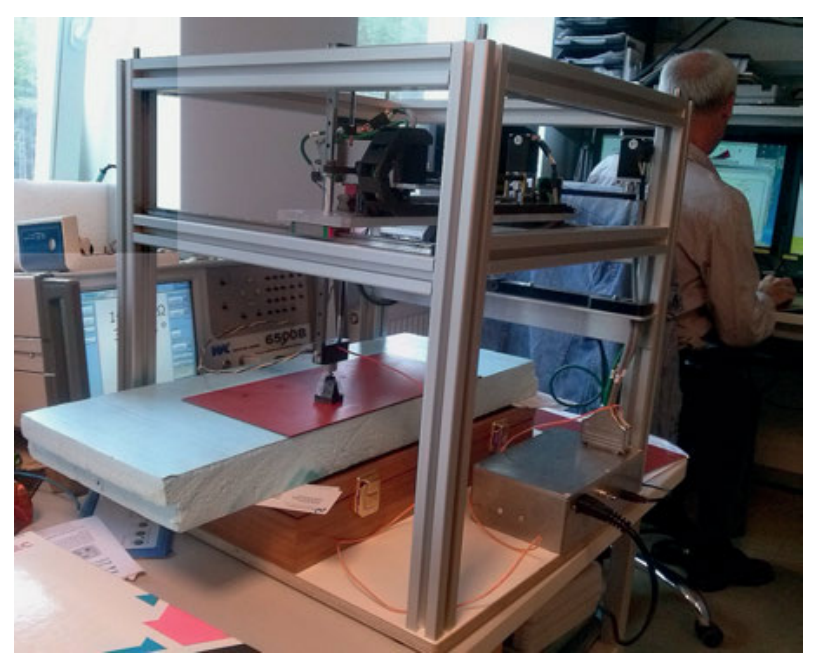

Fig. 3: Developed eddy current scanner.

eddy current modeling, using a combination of the forward model implemented as $\mathrm{C} / \mathrm{C}++$ code in the form of DLL and R-language for reverse solving, analysis and plotting of the features of the impedance measurement results. The single coil above the single-layer metal with variable lift-off and electrical conductivity and/or magnetic permeability approach has been used in this modeling and analysis software.

\subsection{Experiments with initial specimen}

All specimens used in the current research have been produced from the steel EN 10130 class DC01 (similar to the American AISI 1008 grade). On the first specimen (Fig. 4) some round-shape areas were corroded and the whole plate was painted afterwards. The region no. 2 was scanned by the measurement coil. The inverse-modeled results of the apparent relative magnetic permeability $(\mu \mathrm{r})$ at $400 \mathrm{kHz}$, as given in Fig. 5 , show that the corroded part is clearly distinguishable. Also, one profile line has been taken for the lift-off plots, of the same region under interest, shown as a brown vertical line in Fig. 5. Lift-off has been taken for four scans on different lift-off values, shifted vertically by $50 \mu \mathrm{m} / \mathrm{s}$ from each other, as shown on an upper plot of Fig. 6 (a). The same four lift- 

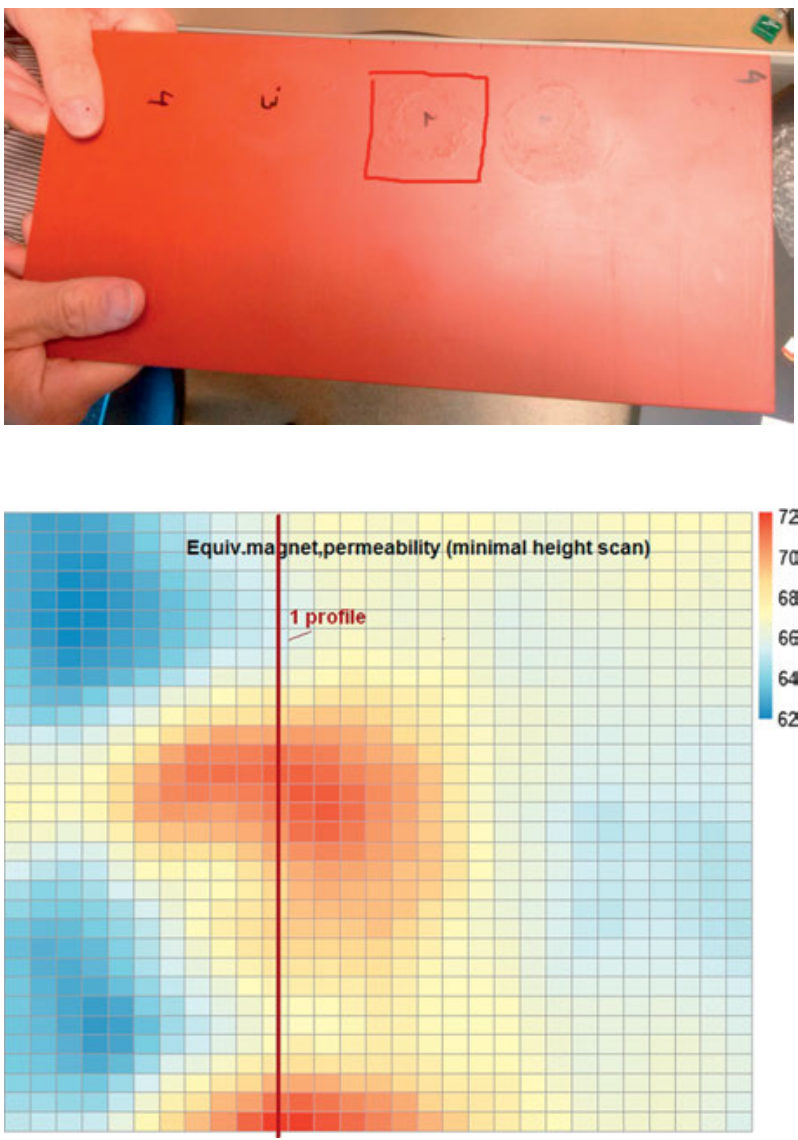

Fig. 4: The initial specimen.

Fig. 5: Apparent relative magnetic permeability of region no. 2 at $400 \mathrm{kHz}$.

off profiles, but shifted vertically onto each other, are shown on the lower plot on the same Fig. 5. The quite exact matching of these four curves indicates the validity of the eddy current modeling and confirms the hypothesis that the corrosion depth can be characterized as change of the apparent lift-off of the material (carbon steel), in the total range of $55 \mu \mathrm{m} / \mathrm{s}$ for the current example.

\subsection{Experiments with specimens no. 1 and 2}

Two specimens have been prepared for the experimental research (no. 1 and no. 2), as shown before and after painting in Fig. 7 and Fig. 8, with round- and strip-shaped corrosion sub-areas, correspondingly. It was noticed that, if using the described air-core planar coil, the resolution of determining of the shape and area of the corroded (rust) area is limited. Still, as seen from Fig. 9, for specimen no. 2, a more or less reasonable corrosion image is acquired. 

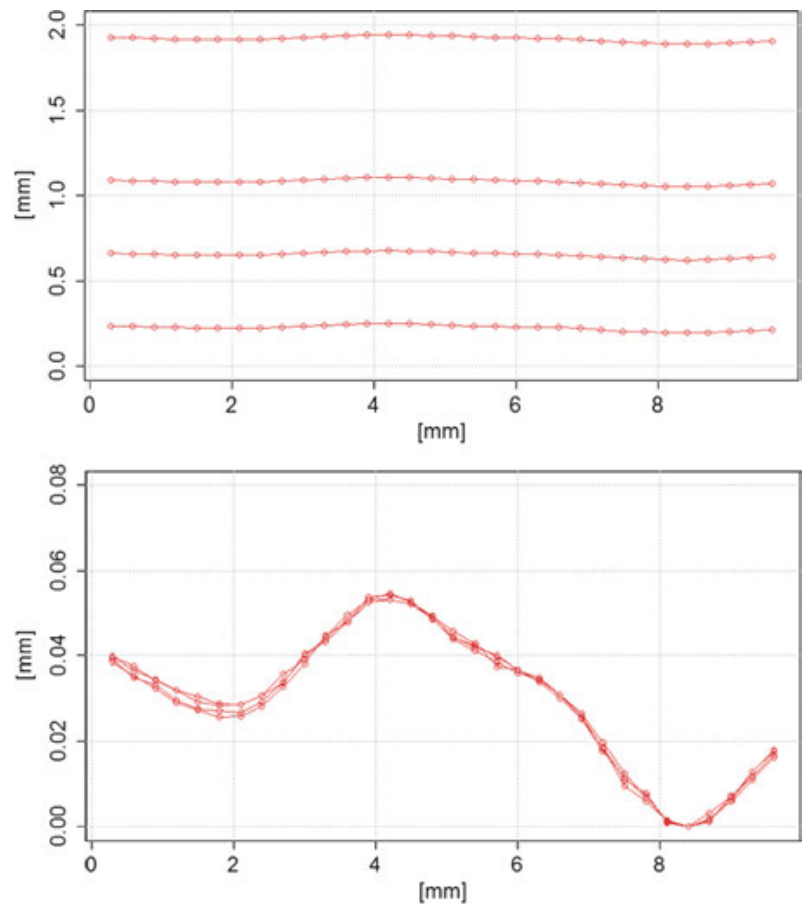

Fig. 6: Four lift-off profiles, inversely calculated from the measurements at $400 \mathrm{kHz}$; absolute lift-off values on the upper plot and relative values on the lower plot.
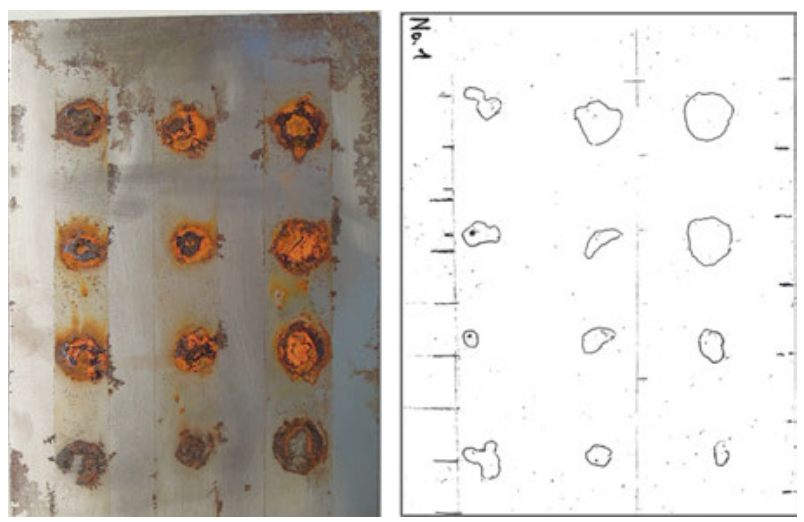

Fig. 7: Specimen no. 1, before and after painting.

\section{Application to estimate the rust area}

To achieve better resolution in the estimation of the rust area shape and size, a magnetic-core measurement coil (diameter of the core is $1.5 \mathrm{~mm}$ and $N=50$ turns) has been introduced (Fig. 10) to work together with the mentioned 3D scanner. As, differently from the air-core approach, in real life it is hard to produce a precise eddy current model, due to several uncertainties (magnetic and geometrical properties of the coil, magnetic core, etc.) simply a complex impedance change has been taken into ac- 

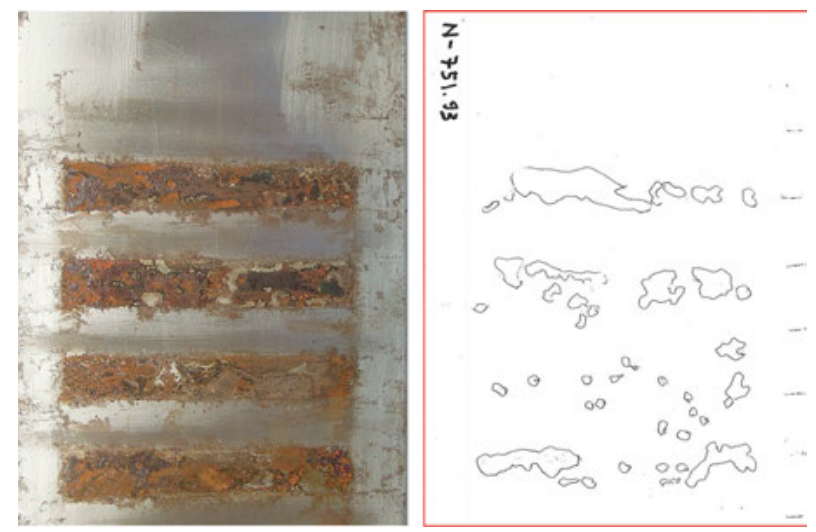

Fig. 8: Specimen no. 2, before and after painting.

\section{Whitef $=400 \mathrm{KHz}$ Muu.png}
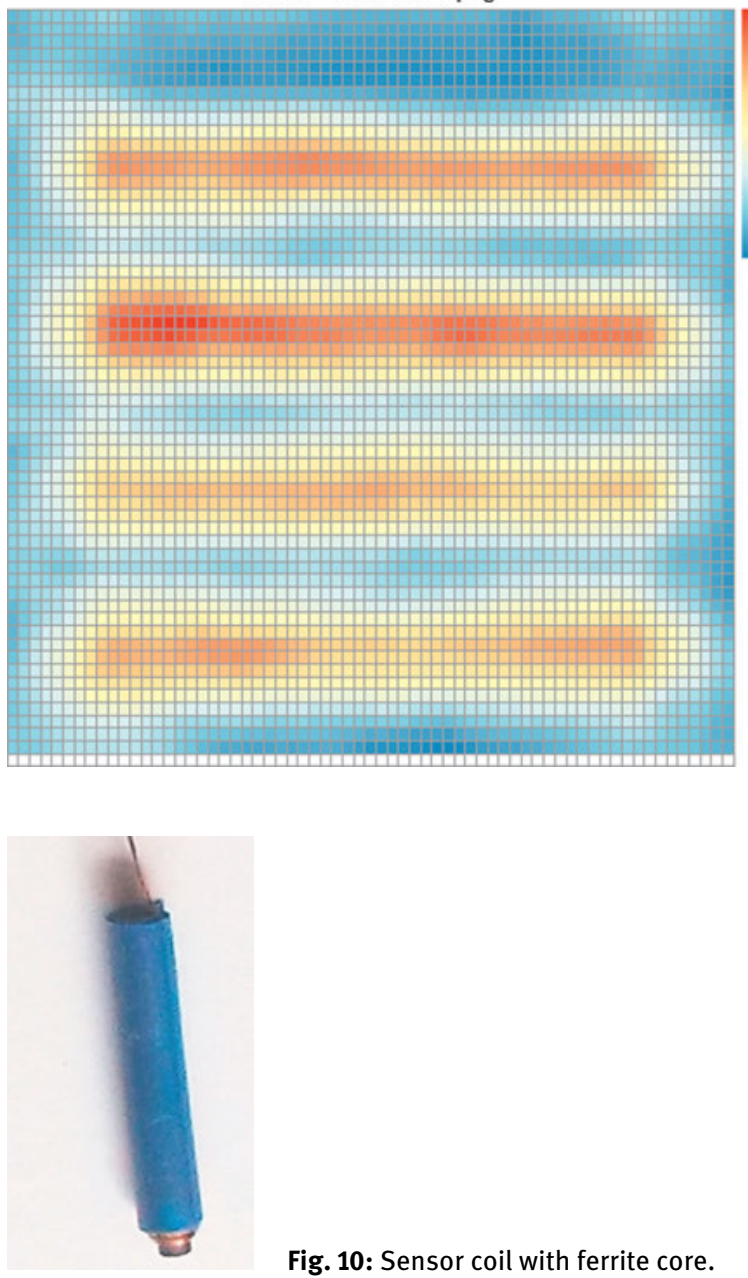

Fig. 10: Sensor coil with ferrite core.

Fig. 9: Apparent magnetic permeability at $400 \mathrm{kHz}$ of specimen no. 2 . 
count for rust measurement. Experimentally it has been found, for the current setup and for a working frequency of $400 \mathrm{kHz}$, the linear combination of the real and imaginary components of the impedance of the coil can be used. This combined impedance values are scanned in 2D at fixed lift-off and acquired as the 2D data array; further the specially developed PC Windows-based application is segmenting this 2D plot as "corroded" and "non-corroded" parts, by selectable threshold, showing the resultant image on the screen (Fig. 11 and Fig. 12). Additionally the user can mark the rectangular region of interest on the image, where the area of the corroded segment is calculated and displayed, in absolute $\left(\mathrm{mm}^{2}\right)$ and relative (\%) units.

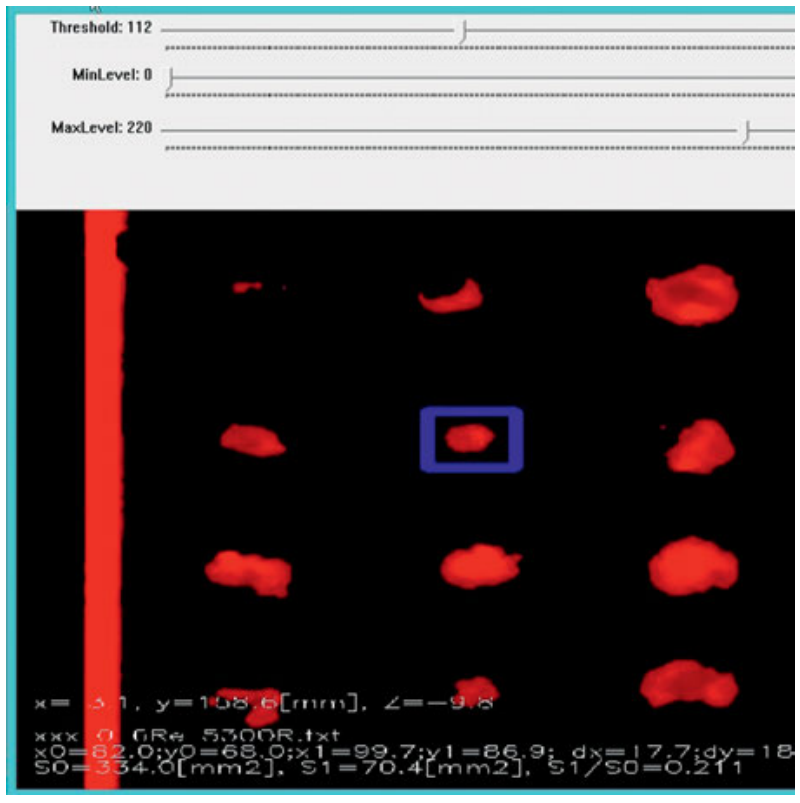

Fig. 11: Windows application for rust area measurement: specimen no. 1.

\section{Conclusion and future work}

The corrosion of metals, specifically the corrosion of the carbon steel, can be efficiently detected and measured by the eddy current approach with single-coil setup, also under a paint layer or similar coatings.

The proposed work is similar to [19], but instead of a pulsed sensor signal the harmonic excitation has been used, with a single, selectable frequency at a time. Also, the usage of the air-core coil in combination with the Dodd-Deeds model enables to get rid of dependency on the sensor lift-off effect and also to estimate directly, without special calibration, the depth of the corrosion. 

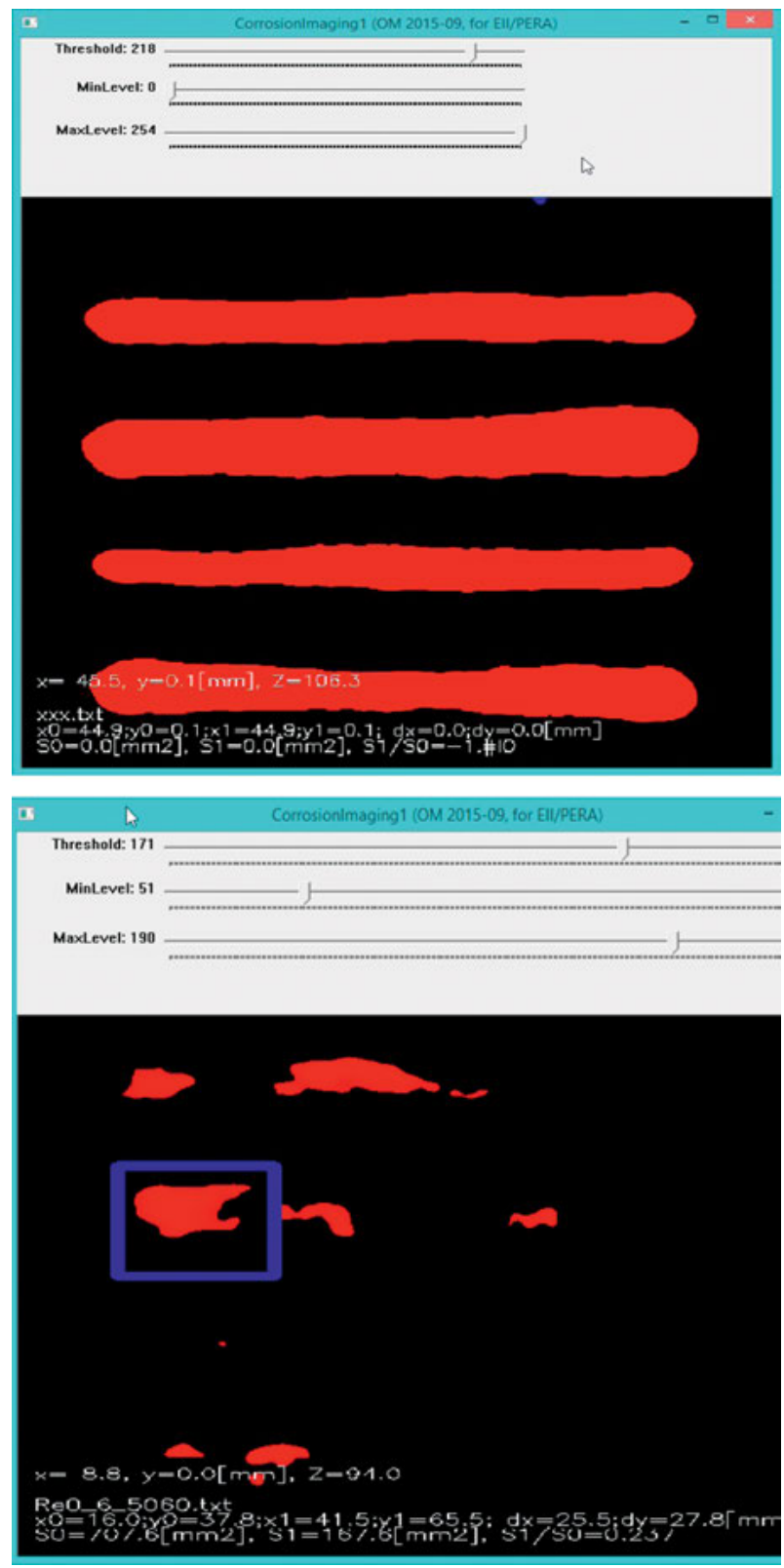

Fig. 12: Windows application for rust area measurement: specimen no. 2, at lower (left image) and higher (right image) thresholds.

The measure of the corrosion can be the variation of the apparent lift-off or change in the apparent magnetic permeability of the material under test. A resolution of $1 \mu \mathrm{m}$ of the apparent lift-off variation can be achieved. A simple Windows application was implemented to measure the area of the corroded part(s). Further low-cost handhold instrumentation can be developed for such measurements. One future re- 
search challenge is to develop the improved absolute models for eddy current-based corrosion measurement. Also, fusion of larger planar air-core coils with smaller ferrite core probes into the same measurement procedure can combine the absolute (model-based) accuracy of the first and good spatial resolution of the second.

\section{Bibliography}

[1] J. García-Martín, J. Gómez-Gil, and E. Vázquez-Sánchez, “Non-destructive techniques based on eddy current testing”, Sensors, vol. 11, no. 3, pp. 2525-2565, 2011.

[2] C. Dodd and W. Deeds, "Analytical solutions to eddy-current probe-coil problems", Journal of Applied Physics, vol. 39, no. 6, pp. 2829-2838, 1968.

[3] C. V. Dodd and W. E. Deed, "Calculation of Magnetic Fields from Time-Varying Currents in the Presence of Conductors”, NASA STI/Recon Technical Report N, vol. 76, Jul. 1975.

[4] S. K. Burke and M. E. Ibrahim, "Mutual impedance of air-cored coils above a conducting plate", Journal of Physics D, Applied Physics, vol. 37, no. 13, pp.1857-1868, 2004.

[5] C. Dodd and W. Deeds, "Absolute Eddy-Current Measurement of Electrical Conductivity", Oak Ridge National Lab., TN (USA), Tech. Rep., 1981.

[6] O. Martens, R. Land, R. Gordon, M. Min, M. Rist, and A. Pokatilov, "Precise eddy current measurements: Improving accuracy of determining of the electrical conductivity of metal plates", In: Lecture Notes on Impedance Spectroscopy: Measurement, Modeling and Applications, vol. 4, 2014, pp.109-115.

[7] M. Parker, A. Pokatilov, T. Kubarsepp, O. Martens, and A. Kolyshkin, "Investigation of planar coil for eddy current conductivity measurements in wide frequency range", In: 19th IMEKO TC4 Symposium - Measurements of Electrical Quantities 2013 and 17th International Workshop on ADC and DAC Modelling and Testing, 2013, pp.55-59.

[8] A. Kolyshkin, T. Kubarsepp, O. Martens, and A. Pokatilov, "Determination of electrical conductivity of metal plates using planar spiral coils", In: Proceedings of the 13th WSEAS International Conference on Systems - Held as part of the 13th WSEAS CSCC Multiconference, 2009, pp. 545-550.

[9] T. P. Theodoulidis and M. Kotouzas, "Eddy current testing simulation on a personal computer", In: 15th World Conference on Nondestructive Testing, Roma (Italy), 15-21 October 2000, Retrieved 26 May 2016, 2002. [Online]. Available: http://www.ndt.net/article/wcndt00/ papers/idn228/idn228.htm.

[10] T. Theodoulidis and E. Kriezis, "Series expansions in eddy current nondestructive evaluation models”, Journal of Materials Processing Technology, vol.161, no. 1-2, pp. 343-347, 2005.

[11] M. Fan, B. Cao, P. Yang, W. Li, and G. Tian, "Elimination of liftoff effect using a model-based method for eddy current characterization of a plate", NDT and E International, vol. 74, pp. 66-71, 2015.

[12] A. Pokatilov, M. Parker, T. Kübarsepp, O. Märtens, and A. Kolyshkin, "Grid-based computational algorithm for accurate ac conductivity measurements", In: 16th International Congress of Metrology, EDP Sciences, 2013, p. 11007.

[13] S. Norton and J. Bowler, "Theory of eddy current inversion", Journal of Applied Physics, vol. 73, no. 2, pp. 501-512, 1993.

[14] J. H. Rose, E. Uzal, and J. C. Moulder, "Magnetic permeability and eddy-current measurements", In: Review of Progress in Quantitative Nondestructive Evaluation, Springer, 1995, pp. 315-322. 
[15] Y. Nonaka, "A double coil method for simultaneously measuring the resistivity, permeability, and thickness of a moving metal sheet", IEEE Transactions on Instrumentation and Measurement, vol. 45, no. 2, pp. 478-482, Apr 1996.

[16] X. Ma, A. Peyton, and Y. Zhao, "Eddy current measurements of electrical conductivity and magnetic permeability of porous metals", NDT and E International, vol. 39, no. 7, pp. 562-568, 2006.

[17] N. Bowler, "Frequency-dependence of relative permeability in steel", In: AIP Conference Proceedings, vol. 820 II, 2006, pp. 1269-1276.

[18] Y. Gotoh, H. Hirano, M. Nakano, K. Fujiwara, and N. Takahashi, "Electromagnetic nondestructive testing of rust region in steel”, IEEE Transactions on Magnetics, vol.41, no. 10, pp. 3616-3618, 2005.

[19] Y. He, G. Tian, H. Zhang, M. Alamin, A. Simm, and P. Jackson, "Steel corrosion characterization using pulsed eddy current systems”, IEEE Sensors Journal, vol. 12, no. 6, pp. 2113-2120, 2012. 